\title{
PNEUMOTÓRAX Pós-ACUPUNTURA: APRESENTAĢÃO CLÍNICA E TRATAMENTO
}

\author{
Ricardo Mingarini Terra*, Angelo Fernandez, Ricardo Helbert Bammann, Ana Cristina P. Castro, Augusto Ishy, Jader Joel Machado \\ JUNQUEIRA \\ Trabalho foi realizado no Núcleo Avançado de Tórax do Hospital Sírio-Libanês - São Paulo, SP
}

\author{
*Correspondência \\ Rua Adma Jafet, 50 - ci 55 - \\ Cerqueira César \\ São Paulo/ SP \\ Cep 01308050 \\ Tel./Fax: (11) 3214-6661 \\ rmterra@vol.com.br
}

\begin{abstract}
RESUMO
Oвjetivos. Apesar de raro, o pneumotórax é um evento adverso da acupuntura potencialmente grave. Visto sua baixa freqüência, apenas relatos de casos são disponíveis e faltam informações quanto os seus aspectos clínicos e terapêuticos. O objetivo deste trabalho é avaliar apresentação clínica, tratamento e evolução do pneumotórax pós-acupuntura.

Métodos. Análise retrospectiva de pacientes com pneumotórax pós-acupuntura tratados em hospital terciário no período $2001-2006$

Resultados. Cinco pacientes (Três homens e duas mulheres) com idade média de 46 anos (30 - 73) foram incluídos. Com exceção de um caso em que houve pneumotórax bilateral, em todos houve perfuração pleural à esquerda. Dor torácica foi o sintoma inicial em todos os pacientes, sendo intensa em três casos, levando a procura imediata por serviço de emergência; e leve em dois casos. Quatro pacientes foram tratados com drenagem pleural (três imediatamente após a admissão e uma após falha de tratamento conservador), devido à sintomatologia exuberante e/ou volume do pneumotórax, e um paciente foi tratado conservadoramente. Todos os pacientes apresentaram evolução clínica satisfatória após resolução do pneumotórax e, em seguimento de 6 meses após o evento, apresentavam-se assintomáticos e sem alterações significativas à radiografia de tórax.

Conclusão. Dor torácica ocorreu em todos os casos, em intensidade variável; a drenagem pleural foi a terapêutica mais frequentemente instituída; e todos os casos evoluíram satisfatoriamente, sem complicações.

UnITERMOS: Terapia por acupuntura. Doença iatrogênica.
\end{abstract}

\section{INTRODUÇÃO}

Tratamentos por acupuntura são difundidos entre médicos e pacientes como procedimentos seguros e sem complicações, porém a introdução das agulhas não é isenta de riscos'. Várias publicações relatam sérias lesões associadas à acupuntura, inclusive com desfecho fatal, como casos de tamponamento cardíaco e pneumotórax bilateral ${ }^{2-4}$. Dentre as diversas complicações, 0 pneumotórax é o descrito com maior freqüência. A inserção de agulhas em região paraesternal, supra ou infraclavicular, paravertebral e porção lateral do tórax para tratamento de dores crônicas, por exemplo, podem provocar perfuração pleural e pulmonar ${ }^{5-7}$. Ainda que seja considerado uma das mais freqüentes complicações pós-acupuntura, o pneumotórax é raro, sendo que na literatura brasileira apenas dois casos foram relatados ${ }^{8,9}$.

Apesar de raro, o pneumotórax é um evento adverso potencialmente grave. Visto sua baixa freqüência, apenas relatos de casos são disponíveis e faltam informações quanto seus aspectos clínicos e terapêuticos. O objetivo deste trabalho é avaliar apresentação clínica, tratamento e evolução do pneumotórax pós-acupuntura.

\section{Métodos}

Análise retrospectiva de prontuários de pacientes com diagnóstico de pneumotórax pós-acupuntura atendidos em nossa Instituição (hospital terciário) no período 2001 - 2006. O trabalho foi avaliado e autorizado pela Comissão de Ética Institucional, e o consentimento informado foi considerado dispensável devido seu desenho retrospectivo.

O diagnóstico do pneumotórax foi baseado em critérios clínicos, dor pleurítica e/ou dispnéia e confirmado por radiografia de tórax demonstrando claramente a linha do pneumotórax. A relação com acupuntura foi estabelecida da seguinte forma: pacientes que foram submetidos à sessão (ou sessões) de acupuntura na região torácica em no máximo 10 dias antecedendo o diagnóstico do pneumotórax. Para evitar fatores de confusão, não foram considerados pacientes com histórico de pneumotórax prévio, antecedente recente $(<60$ dias $)$ de trauma, cirurgia ou procedimentos invasivos e dor torácica aguda recente $(<15$ dias) como motivo da acupuntura.

A conduta estabelecida foi drenagem pleural em caso de sintomas refratários à terapêutica clínica inicial (oxigênio e analgesia) e/ ou radiografia demonstrando pneumotórax ocupando mais de $20 \%$ do hemitórax. Pacientes que não se enquadraram nestes critérios receberam tratamento conservador, baseado em analgesia e controle radiológico (em 6 horas e 48 horas). Em todos os casos drenados, foi utilizado cateter pleural de $14 \mathrm{Fr}$ acoplado a válvula unidirecional de Heimlich, introduzido sob anestesia local no segundo espaço intercostal linha hemiclavicular ou sexto espaço intercostal linha axilar média (se houver presença concomitante de líquido). O dreno foi mantido até expansão pulmonar completa confirmada por radiografia de tórax, débito inferior a $200 \mathrm{~mL} / 24 \mathrm{~h}$ 


\begin{tabular}{|c|c|c|c|c|c|}
\hline \multicolumn{6}{|c|}{ Tabela I - Resumo dos casos de pneumotórax iatrogênico após acupuntura } \\
\hline & Caso I & Caso 2 & Caso 3 & Caso 4 & Caso 5 \\
\hline $\begin{array}{l}\text { Sexo } \\
\text { Idade } \\
\text { Indicação da acupuntura }\end{array}$ & $\begin{array}{l}\text { Masculino } \\
\text { 30anos } \\
\text { Cérvico-lombalgia }\end{array}$ & $\begin{array}{l}\text { Feminino } \\
73 \text { anos } \\
\text { Lombalgia }\end{array}$ & $\begin{array}{l}\text { Feminino } \\
42 \text { anos } \\
\text { Tóraco-lombalgia }\end{array}$ & $\begin{array}{c}\text { Masculino } \\
45 \text { anos } \\
\text { Hérnia de Disco Cervical }\end{array}$ & $\begin{array}{l}\text { Masculino } \\
40 \text { anos } \\
\text { Lombalgia }\end{array}$ \\
\hline $\begin{array}{l}\text { Tempo até procura por atendimento } \\
\text { especializado }\end{array}$ & Imediato & Imediato & 5 dias & Imediato & 2dias \\
\hline $\begin{array}{l}\text { Hemitórax afetado } \\
\text { Conduta } \\
\text { Tempo de drenagem }\end{array}$ & $\begin{array}{c}\text { HTE } \\
\text { Drenagem Pleural E } \\
3 \text { dias }\end{array}$ & $\begin{array}{c}\text { BIL } \\
\text { Drenagem Pleural E, Conservadora D } \\
\text { 3 dias }\end{array}$ & $\begin{array}{c}\text { HTE } \\
\text { Conservadora }\end{array}$ & $\begin{array}{c}\text { HTE } \\
\text { Drenagem Pleural } \\
3 \text { dias }\end{array}$ & $\begin{array}{c}\text { HTE } \\
\text { Drenagem Pleural } \\
\text { 3 dias }\end{array}$ \\
\hline
\end{tabular}

HTD - hemitórax direito; HTE-hemitórax esquerdo; BIL-bilateral; E-esquerda; D-direita.

e ausência de fístula aérea por tempo superior a 24 horas.

\section{Resultados}

Cinco pacientes (Três homens e duas mulheres) com idade média de 46 anos (variando entre 30 - 73 anos) foram incluídos. Com exceção de um caso em que houve pneumotórax bilateral, em todos houve perfuração pleural à esquerda. A Tabela I apresenta um resumo dos casos.

Todos os cinco pacientes procuraram tratamento por meio da acupuntura para dores osteomusculares crônicas no dorso (porção cervical e torácica), e os pontos de punção foram nas regiões paravertebrais e subescapulares.

Em três pacientes, o aparecimento de dor torácica de forte intensidade foi imediatamente relacionado à sessão de acupuntura, implicando atendimento médico imediato. Dois foram submetidos à drenagem pleural imediata devido aos sintomas (dispnéia leve e dor torácica intensa) e volume do pneumotórax. Um destes, o paciente com pneumotórax bilateral, teve o hemitórax esquerdo drenado, porém não foi necessária intervenção à direita, visto o pneumotórax ter sido considerado pouco volumoso deste lado. 0 terceiro paciente foi inicialmente tratado de forma conservadora, porém necessitou de drenagem pleural seis horas após a admissão hospitalar, por progressão do pneumotórax.

Dois pacientes procuraram atendimento médico num período superior a 48 horas após a sessão de acupuntura. A principal queixa de ambos foi dor torácica de pequena intensidade, que inicialmente foi confundida pelos pacientes com os sintomas que os fizeram procurar a acupuntura. A persistência da dor foi o fator determinante para que ambos procurassem por atendimento especializado. Um destes foi submetido à drenagem imediata e o outro paciente foi tratado conservadoramente (cinco dias já haviam passado após a acupuntura e o mesmo encontrava-se oligossintomático).

Nenhuma complicação ocorreu durante as quatro drenagens pleurais (três imediatamente após a admissão e uma após falha de tratamento conservador) realizadas nesta série e todos os drenos foram retirados no terceiro dia pós-drenagem sem intercorrências. Três pacientes foram submetidos a tratamento conservador (um deles tinha pneumotórax bilateral, o outro hemitórax foi drenado), sendo que houve uma falha devido à progressão do pneumotórax, conforme mencionado previamente. Os outros dois casos tiveram

\section{Figura I - Superior: agulha utilizada habitualmente para acupuntura; Inferior: agulha $30 \times 7 \mathrm{~mm}$ habitualmente utilizada em ambiente hospitalar}

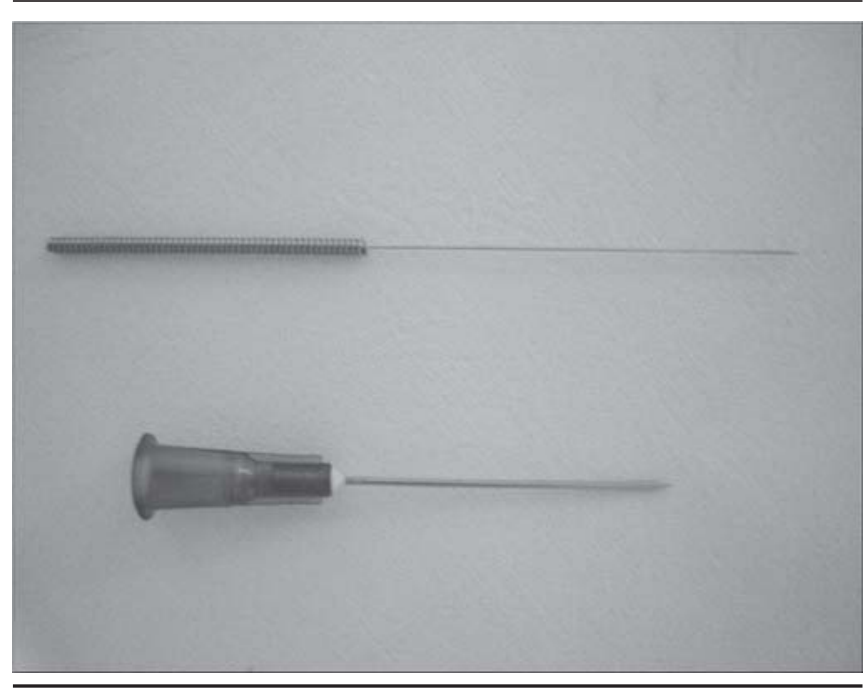

boa evolução, com redução do pneumotórax confirmada em radiografia após 48 horas e absorção total após I mês.

Todos os cinco pacientes apresentaram evolução clínica satisfatória após a resolução do episódio de pneumotórax e em seguimento de seis meses após o evento apresentavam-se assintomáticos e sem alterações significativas à radiografia de tórax.

\section{Discussão}

O tratamento por acupuntura consiste na inserção de agulhas de 15 a $50 \mathrm{~mm}$ de comprimento através da pele, com profundidade variando de poucos milímetros a alguns centímetros. Na maioria dos casos, a agulha atinge exclusivamente planos músculoesqueleticos, mas algumas vezes, penetrando além do limite, pode atingir outras estruturas, incluindo nervos ou vasos, além de estruturas viscerais profundas, como a pleura, peritônio ou o pericárdio ${ }^{2}$. Um estudo anatômico, em cadáveres humanos a fresco, mostrou que punções com profundidade de 1 a $2 \mathrm{~cm}$, tanto na linha paraesternal quanto na hemiclavicular, podem atingir o pulmão ${ }^{2}$. Para esta avaliação, os cadáveres foram congelados e submetidos a cortes transversais, permitindo assim a análise das diferentes distâncias entre a superfície da pele e as estruturas 
Tabela 2- Casos de pneumotórax iatrogênico pós-acupuntura presentes na literatura de língua inglesa e portuguesa e publicados a partir do ano de 1990

\begin{tabular}{|c|c|c|c|c|c|}
\hline Autores & Hemitórax & Tempo até sintomas* & Sintomas/Sinais & Tratamento (tempo de drenagem) & Evolução \\
\hline Su IW, etal. $(2007)^{13}$ & Bilateral & Imediato & Dispnéia & \multirow{10}{*}{\multicolumn{2}{|c|}{$\begin{array}{cc}\text { Drenagem pleural } \mathrm{E}(2 \text { dias })+\text { Conservador D } & \text { Assintomática } \\
\text { Drenagem Pleural D }(2 \text { dias }) & \text { Assintomática } \\
\text { Drenagem Pleural Bilateral (4 dias) } & \text { Assintomática } \\
\text { Conservador } & \text { Assintomático } \\
\text { Drenagem Pleural E }(7 \text { dias }) & \text { Assintomática } \\
\text { Drenagem Pleural Bilateral } & \text { Óbito*** } \\
\text { Drenagem Pleural E } & \text { Assintomático } \\
\text { Conservador } & \text { Assintomática } \\
\text { Drenagem Pleural D } & \text { Assintomática } \\
\text { Punção Pleural } & \text { Assintomático }\end{array}$}} \\
\hline Chauffe RJ, etal $(2006)^{14}$ & Direito & Imediato & Dor Torácica, Dispnéia e Tosse & & \\
\hline Lee WM, etal. $(2005)^{\prime}$ & Bilateral & Imediato & DorTorácica & & \\
\hline Saifekdeen K, et al. $(2004)^{15}$ & Direito & Imediato & Dispnéia e Dor Torácica & & \\
\hline PeukerE, etal. $(2004)^{16}$ & Esquerdo & Imediato & Dispnéia e Dor Torácica & & \\
\hline Iwadate $K$, etal. $(2003)^{4}$ & Bilateral & Imediato & Dispneia e Dor Torácica & & \\
\hline Leung $/ S$, etal. $(2002)^{17}$ & Esquerdo & Imediato & Dispnéia & & \\
\hline Kao CL, etal. $(2002)^{18}$ & Bilateral & 粎 & Ausentes & & \\
\hline \multirow{2}{*}{ Vilke GM, etal.(1997)6 } & Direito & Imediato & Dispnéia e Dor Torácica & & \\
\hline & Esquerdo & Imediato & Dispnéia e Dor Torácica & & \\
\hline CricentiSV, etal. (I996) ${ }^{9}$ & Esquerdo & Imediato & Dispnéia e Dor Torácica & Drenagem Pleural E & Assintomática \\
\hline Willms D, etal. $(|99|)^{19}$ & Bilateral & Imediato & Dispnéia, Hipotensão, Taquicardia & Drenagem Pleural Bilateral (alguns dias) & Assintomático \\
\hline Wright RS, etal. (|99|20 & Bilateral & Imediato Dispn & néia, Dor Torácica, Hipotensão e Taquicardia & Drenagem Pleural Bilateral (9 dias) & Assintomática \\
\hline Morrone $N$, etal. $(1990)^{8}$ & Esquerdo & Imediato & Dispnéia e Tosse seca & Drenagem Pleural E (3 dias) & Assintomático \\
\hline
\end{tabular}

* Foi considerado "imediato" quando o tempo até o surgimento dos sintomas era d" I hora;

***óbito ocorreu 90 minutos após aadmissão da pacienteno serviço de emergência;

**** Estas informações não estão disponíveis na publicação. E-esquerda, D-Direita.

anatômicas atingidas. De acordo com a literatura, a inserção de agulhas em região paraesternal (KI22 a KI27), supraclavicular (GB2I, STII e STI2) ou infraclavicular (LU2, STI3 e KI27), hemiclavicular (STI2 a STI8) e escapular média (BL4I a BL50) requer um cuidado particular, já que pode provocar perfuração pleural e pulmonar ${ }^{1,5-7}$. O sistema de nomenclatura dos pontos de acupuntura citados acima é baseado no "World Health Organization Regional Working Group on the Standardization of Acupuncture Nomenclature"10. A Figura I mostra a agulha utilizada em um dos casos de nossa série; é interessante notar que esta é significativamente mais longa que a agulha $30 \times 7 \mathrm{~mm}$, habitualmente utilizada para toracocentese diagnóstica.

A ocorrência de pneumotórax pós-acupuntura é rara, tendo sido observado, em revisão sistemática, um evento a cada 125 mil sessões ${ }^{\prime \prime}$, e, em estudo observacional prospectivo, dois casos em 97.733 pacientes submetidos a acupuntura'2. Ainda assim, em uma revisão recente de artigos reportando eventos adversos relacionados a acupuntura (incluindo relatos de caso, séries de casos e outras formas de publicação), foram contabilizados 715 casos, destes, 191 foram pneumotórax, 17 resultando em morte 3 . Infelizmente, neste estudo, não foram apresentados detalhes quanto as características dos pacientes ou evolução clínica. A baixa freqüência dos eventos talvez justifique a ausência de séries de casos na literatura, uma vez que apenas relatos de até três casos foram identificados em nossa revisão bibliográfica. A Tabela 2 resume os casos descritos na literatura de língua inglesa e portuguesa, publicados a partir de 1990, e que ofereciam as informações clínicas necessárias para análise comparativa com nossa série. Uma observação interessante é a maior ocorrência de pneumotórax no hemitórax esquerdo, fato verificado tanto em nossa casuística, quanto nos casos previamente relatados (Tabela 2). Não encontramos argumentos anatômicos ou clínicos para justificar esta observação, e uma maior amostra é necessária para confirmar tal dado.
Nesta série, a dor torácica ocorreu em todos os pacientes, sintoma mais frequentemente observado também nos relatos de caso analisados $1,4,6,8,9,13-20$. Porém, sua intensidade foi variada, tendo sido bem evidente em três casos, o que levou a procura imediata por serviço de emergência. Nos demais, os sintomas foram menos expressivos, podendo ser confundidos com os sintomas que fizeram os pacientes procurarem a acupuntura, fato já notificado previamente na literatura ${ }^{6,8,16,17}$. Este tipo de situação reforça a possibilidade de subnotificação e gera atrasos no diagnóstico, especialmente se o grau de suspeita clínica for baixo.

O tratamento do pneumotórax pós-acupuntura é semelhante ao tratamento de pneumotórax iatrogênico de outras etiologias: observação clínica ou drenagem pleural, conforme volume do pneumotórax e quadro clínico do paciente. Assim como nos casos relatados na literatura (Tabela 2 ), a maioria dos pacientes foi submetida a drenagem pleural com boa evolução.

Infelizmente, o pequeno número de pacientes e o desenho retrospectivo limitam a força de nossas conclusões. Porém, o pequeno número de pacientes nesta série reflete a raridade desta complicação, o que também praticamente impossibilita a realização de estudos prospectivos a respeito do tema.

O pneumotórax é uma rara e pouco divulgada complicação da acupuntura. Seu conhecimento, principalmente por aqueles que praticam e indicam o método, é fundamental por se tratar de um evento potencialmente grave e de apresentação clínica muitas vezes pouco exuberante. $\bigcirc$ quadro clínico inicial mais freqüente é a dor torácica imediatamente após a sessão de acupuntura, que pode ser de intensidade variável. A drenagem pleural, apesar de reservada para pacientes sintomáticos e/ou com pneumotórax volumoso, é a terapêutica mais frequentemente indicada. Nesta série, todos os pacientes tiveram boa evolução, sem complicações adicionais. 
Terra RM et al.

Conflito de interesse: não há.

\section{SUMMARY}

\section{PNeumothoraX after ACUPUNCTURE: Clinical PRESENTATION AND MANAGEMENT}

INTRODUCTION. Pneumothorax is a rare but dangerous complication of acupuncture. Because of its rarity, there are few reports in literature and, therefore little information regarding clinical and therapeutic aspects. This article aims to analyze the clinical presentation, management and follow-up of patients with pneumothorax after acupuncture.

Methods. Retrospective study of patients with postacupuncture pneumothorax evaluated in a tertiary hospital during a five-year period (200I-2006).

RESULTS. Five patients ( 3 male and 2 female), mean age 46 years (30 - 73) were included. All patients but one (who had a bilateral pneumothorax) had left-sided pneumothorax. Chest pain, which was the initial symptom in all patients was severe in three cases and mild in two. Four patients underwent tube thoracostomy (pig-tail catheter), three of them immediately after admission and the other after a failed 12-hour conservative treatment period. One patient had a successful conservative management. All had an excellent outcome and were asymptomatic and exhibited a normal chest $X$ ray at 6-month follow-up

CONCLUSION. In all patients, the initial symptom was chest pain, of varying intensity. Tube thoracostomy was the therapeutic modality most frequently employed. All patients had a successful outcome with no further complications. [Rev Assoc Med Bras 2007; 53(6): 535-8]

KEY wORDS: Pneumothorax. Acupuncture Therapy, latrogenic Disease.

\section{REFERÊNCIAS}

I. Lee WM, Leung HB, Wong WC. latrogenic bilateral pneumothorax arising from acupuncture: a case report. J Orthop Surg. (Hong Kong) 2005; 13:300-2.

2. Peuker ET, White A, Ernst E, Pera F, Filler TJ. Traumatic complications of acupuncture. Therapists need to know human anatomy. Arch Fam Med. 1999;8:553-8.
3. White A. A cumulative review of the range and incidence of significant adverse events associated with acupuncture. Acupunct Med. 2004;22: 1 22-33.

4. Iwadate K, Ito H, Katsumura S, Matsuyama N, Sato K, Yonemura I, et al. An autopsy case of bilateral tension pneumothorax after acupuncture. Leg Med. (Tokyo) 2003; 5:170-4.

5. Ritter HG, Tarala R. Pneumothorax after acupuncture. BMJ. 1978:2(6|37):602-3.

6. Vilke G, Wulfert E. Case reports of two patients with pneumothorax following acupuncture. J Emerg Med. 1997; I5: I55-7.

7. Mazal DA, King T, Harvey J, Cohen J. Bilateral pneumothorax after acupuncture. N Engl J Méd. 1980;302:|365-6.

8. Morrone N, Freire JAS, Ferreira AKA, Dourado AM. Pneumotórax iatrogênico por acupuntura. Rev Paul Med. 1990; |08:|89-9|.

9. Cricenti SV, Yamamura Y, Matsumoto JT, Juliano Y, Esper RS. Pneumotórax iatrogênico por inserção de agulha de acupuntura no triângulo da ausculta. Rev Paul Acupunt. 1996;2:40-3.

I 0. Gongwan L. Acupoints and meridians. Beijing, China: Huaxia Publishing House; 1997.

I I. Ernst E, White AR. Prospective studies of the safety of acupuncture: a systematic review. Am J Med. 200।; | 10:48।-5.

12. Melchart D, Weidenhammer W, Streng A, Reitmayr S, Hoppe A, Ernst $\mathrm{E}$, et al. Prospective investigation of adverse effects of acupuncture in 97733 patients. Arch Intern Med. 2004;164:104-5.

13. Su JW, Lim CH, Chua YL. Bilateral pneumothoraces as a complication of acupuncture. Singapore Med J. 2007;48:e32-3.

14. Chauffe RJ, Duskin AL. Pneumothorax secondary to acupuncture therapy. South Med J. 2006;99:1297-9.

15. Saifeldeen K, Evans M. Acupuncture associated pneumothorax. Emerg Med J. 2004;21:398.

16. Peuker E. Case report of tension pneumothorax related to acupuncture. Acupunct Med. 2004;22:40-3.

17. Leung JS. Complementary medicine, acupuncture, and pneumothorax. Hong Kong Med J. 2002;8:225.

18. Kao CL, Chang JP. Bilateral pneumothorax after acupuncture. J Emerg Med. 2002;22:101-2

19. Willms D. Possible complications of acupuncture. West J Med. | 99|; | 54:736-7.

20. Wright RS, Kupperman JL, Liebhaber MI. Bilateral tension pneumothoraces after acupuncture. West J Med. 1991;154:102-3. 